\title{
Cost-benefit analysis of Xpert MTB/RIF for tuberculosis suspects in German hospitals
}

\author{
Roland Diel ${ }^{1}$, Albert Nienhaus ${ }^{2}$, Doris Hillemann ${ }^{3}$ and Elvira Richter ${ }^{3,4}$
}

Affiliations: ${ }^{1}$ Institute for Epidemiology, University Medical Hospital Schleswig-Holstein, Kiel, Airway Research Center North (ARCN), Member of the German Center for Lung Research, Germany. ${ }^{2}$ Institute for Health Services Research in Dermatology and Nursing, University Medical Center Hamburg-Eppendorf, Hamburg, Germany. ${ }^{3}$ National Reference Center for Mycobacteria, Borstel, Germany. ${ }^{4}$ Labor Limbach, Heidelberg, Germany.

Correspondence: Roland Diel, Institute for Epidemiology, University Medical Hospital Schleswig-Holstein, Niemannsweg 11, 24015 Kiel, Germany. E-mail: roland.dieldepi.uni-kiel.de

ABSTRACT Our objective was to assess the cost-benefit of enhancing or replacing the conventional sputum smear with the real-time PCR Xpert MTB/RIF method in the inpatient diagnostic schema for tuberculosis (TB).

Recent data from published per-case cost studies for TB/multidrug-resistant (MDR)-TB and from comparative analyses of sputum microscopy, mycobacterial culture, Xpert MTB/RIF and drug susceptibility testing, performed at the German National Reference Center for Mycobacteria, were used. Potential cost savings of Xpert MTB/RIF, based on test accuracy and multiple cost drivers, were calculated for diagnosing TB/MDR-TB suspects from the hospital perspective.

Implementing Xpert MTB/RIF as an add-on in smear-positive and smear-negative TB suspects saves on average $€ 48.72$ and $€ 503$, respectively, per admitted patient as compared with the conventional approach. In smear-positive and smear-negative MDR-TB suspects, cost savings amount to $€ 189.56$ and $€ 515.25$ per person, respectively. Full replacement of microscopy by Xpert MTB/RIF saves $€ 449.98$. In probabilistic Monte-Carlo simulation, adding Xpert MTB/RIF is less costly in $46.4 \%$ and $76.2 \%$ of smear-positive TB and MDR-TB suspects, respectively, but $100 \%$ less expensive in all smear-negative suspects. Full replacement by Xpert MTB/RIF is also consistently cost-saving.

Using Xpert MTB/RIF as an add-on to and even as a replacement for sputum smear examination may significantly reduce expenditures in TB suspects.

@ERSpublications

Enhancing/replacing conventional sputum smear testing in TB with Xpert MTB/RIF may result in cost savings http://ow.ly/Stapk 


\section{Introduction}

Although tuberculosis (TB) incidence rates are steadily and globally in decline, multidrug-resistant (MDR)-TB, defined as simultaneous resistance to at least isoniazid and rifampicin (RIF), remains a major public health challenge in the World Health Organization (WHO) European region, where, in 2012, the prevalence of MDR-TB among 90127 new TB cases was 15\% [1]. This trend of increasing MDR-TB prevalence has important health economics implications: 1) it strengthens the long-established requirement of national guidelines that all patients admitted to healthcare facilities with suspected TB be maintained in costly respiratory isolation until it can be assumed that they are no longer contagious [2], and 2) it increases the need for emphasis to be placed on defining and speedily implementing case-appropriate treatment $[3,4]$.

Thus, the rapid diagnosis of TB disease and determination of drug resistance profiles is essential not only for early treatment and the associated prevention of TB transmission, but also highly relevant to the management of scarce economic resources, Since January 1, 2004, hospital costs in Germany have been based on the German Diagnosis Related Group (G-DRG) system, which assigns each TB case to one of two categories (E76B or E76C), depending on the severity of comorbidities. This imposes a fixed "base rate" of payment for 13 days treatment; if hospital treatment of $>13$ days is required (category E76A), the statutory health insurances pay locally negotiated daily rates. In most cases, these fall below the average daily reimbursement for the first 14 days. Accordingly, for TB patients under statutory health insurance, hospitals do well to keep the total number of patients treated high, but to keep the duration of their hospital stays as short as possible $[5,6]$.

The Xpert MTB/RIF test (Cepheid, Sunnyvale, CA, USA) is a real-time PCR assay for simultaneous detection of Mycobacterium tuberculosis complex and of mutations in the rpoB gene that are associated with resistance to RIF as a proxy for MDR-TB from clinical samples. Results are obtained in $2 \mathrm{~h}$ [7]. After having recommended the use of Xpert MTB/RIF in December 2010, the WHO updated its guidance in October 2013, suggesting that the use of Xpert MTB/RIF to diagnose pulmonary TB, paediatric TB, extrapulmonary TB and RIF resistance [8] should be considered. Most recently, the US Food and Drug Administration suggested removing patients with suspected pulmonary TB from airborne infection isolation units after one or two negative Xpert MTB/RIF results. This guidance was based on an in-house clinical validation study that demonstrated negative predictive values (NPVs) of $99.7 \%$ for a single negative acid-fast bacilli (AFB) smear and 100\% for two consecutive negative Xpert MTB/RIF results [9].

As data on the economic impact of implementing Xpert MTB/RIF in low-incidence countries are sparse, we undertook to assess the consequences of routine use of Xpert MTB/RIF with respect to confirmation or exclusion of TB disease and the timing of TB treatment in German TB wards. It is to this setting that the overwhelming majority (78.6\%) of subsequently diagnosed TB cases are primarily admitted [10]. Our aim was to clarify the possible advantages of Xpert MTB/RIF, either performed at the hospital itself or in easy-to-reach local laboratories, as an add-on to conventional smear examinations or alternatively by replacing serial sputum smear microscopy with single-sample Xpert MTB/RIF examination. For our calculations, we used previously unpublished data of the German National Reference Center (NRC) for Mycobacteria in Borstel. Our model was parameterised to receive data on sensitivity, specificity, positive predictive value (PPV) and NPV of Xpert MTB/RIF testing, and to use sputum culture as the reference method. The data used were from untreated TB suspects whose sputa were sent to the NRC between January 1, 2012 and December 31, 2013.

At the NRC, Ziehl-Neelsen microscopy and Xpert MTB/RIF had been performed and one liquid culture (BACTEC MGIT 960; BD, Franklin Lakes, NJ, USA) as well as two solid cultures (Löwenstein-Jensen and Stonebrink TB Medium from NRC production) had been started on the same day. Stratified by smear status, the time (in days) until the first of the cultures became positive and until drug susceptibility testing (DST) results were available was assessed and compared with Xpert MTB/RIF results of the respective patients; negative cultures were uniformly read after 56 days. Culture results were considered as the "gold standard" to which Xpert MTB/RIF results were compared.

\section{Material and methods}

Ethical considerations

Ethical approval was not necessary as only fully anonymised secondary data were used.

\section{Model approach}

The economic analysis included the incremental costs of operating expenditures in diagnosing and treating pulmonary TB suspects for three different strategies. The perspective taken was that of the hospitals themselves, i.e. from admission through to patient discharge. Two Xpert MTB/RIF add-on algorithms and one Xpert MTB/RIF-only algorithm were studied. In the first Xpert MTB/RIF add-on algorithm, all sputum smear-positive individuals were tested with Xpert MTB/RIF on a single sputum specimen and in 
the Xpert MTB/RIF add-on algorithm only smear-negative TB suspects were tested with Xpert MTB/RIF. In the Xpert MTB/RIF-only algorithm, a single sputum specimen was collected for performing Xpert MTB/RIF, followed by a culture; microscopy was not performed.

As hospital costs of the add-on algorithms were calculated separately for each of the two patient groups, i.e. those thought to have fully susceptible TB and those suspected of having MDR-TB, five scenarios were considered (figures 1-5; refer also to online supplementary material).

Full descriptions of the various diagnostic and treatment assumptions of the model are provided in the online supplementary material.

\section{Model structure}

A deterministic, patient-based decision-analytic model was developed simulating the costs of the three approaches as described above for adult German TB and MDR-TB suspects based on German country-specific modalities. We used TreeAge software (TreeAge, Williamstown MA, USA) for model building and analysis. Univariate sensitivity analysis was performed using all variables (with some noted exceptions) to examine the extent to which our calculations were affected by varying selected assumptions. It also revealed the relative importance of the individual variables in each of the five different scenarios. Variation was done at random using either 1) the lower and upper bounds of a parameter's standard deviation or 2) those of its confidence interval. Where these were not applicable, our model simply caused parameter values to vary by $\pm 20 \%$ according to international practice, unless otherwise stated. Furthermore, in order to capture the interactions between multiple inputs we provided a probabilistic sensitivity analysis (PSA) by assigning an appropriate statistical (probability) distribution for all parameters which were randomly drawn in a second-order Monte-Carlo simulation $(n=1000)$. All costs are reported in 2013 Euros $(€)$.

As a result of a lack of valid data, we have not included in our model the effects of transmission by TB or MDR-TB patients to co-patients or healthcare workers.

Input parameters are shown together with their probabilistic distributions in table 3.

\section{Model input}

Laboratory parameters

Laboratory results for Xpert MTB/RIF compared with sputum smears (NRC)

A total of 707 sputa from untreated TB suspects were investigated in the NRC in 2012/2013 with the Xpert MTB/RIF test. Prevalence of TB in that collective proved to be $19.66 \%$ (95\% CI 16.91-22.94).

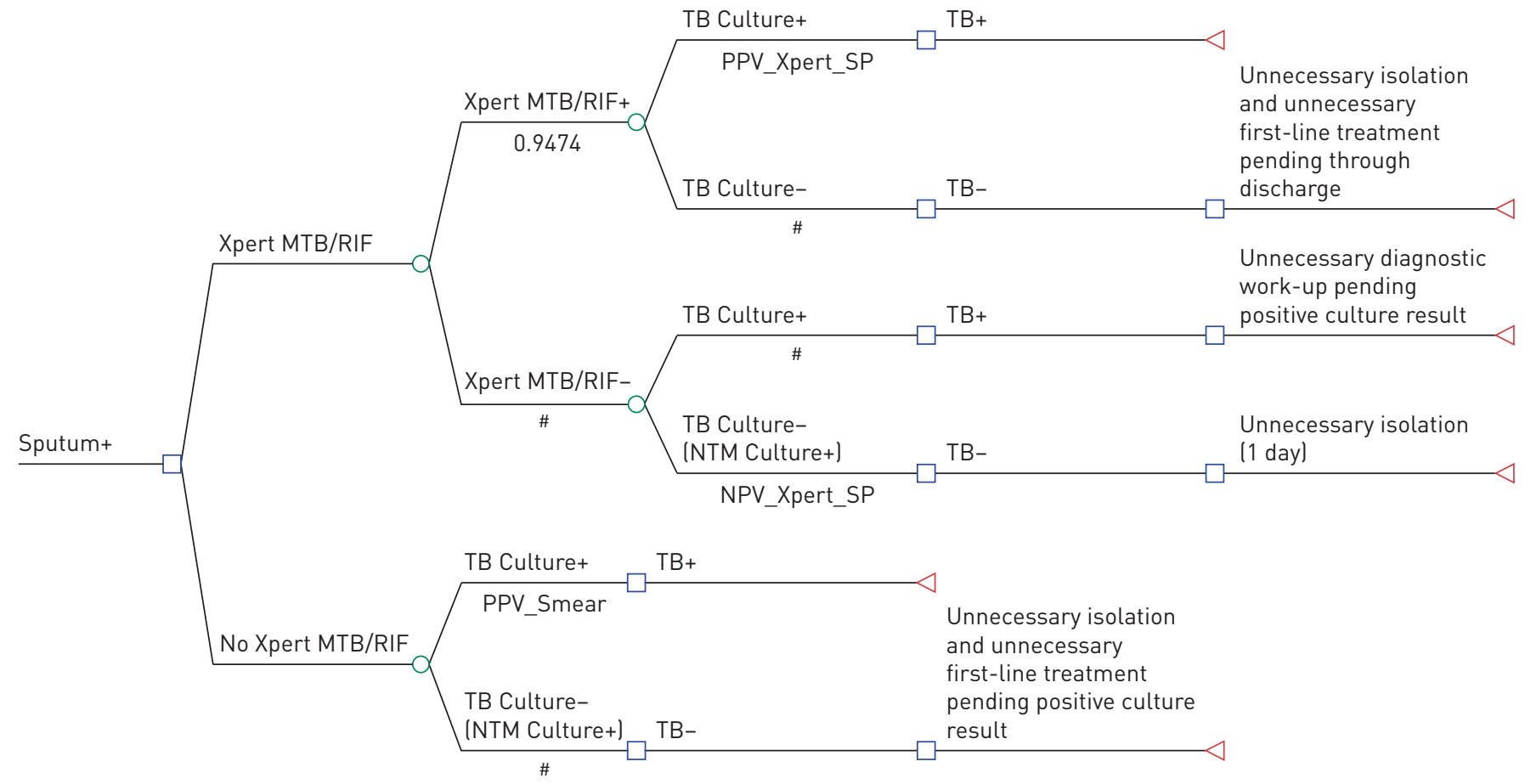

FIGURE 1 Xpert MTB/RIF replacing the sputum-based approach in tuberculosis (TB) suspects. NTM: nontuberculous mycobacteria. 


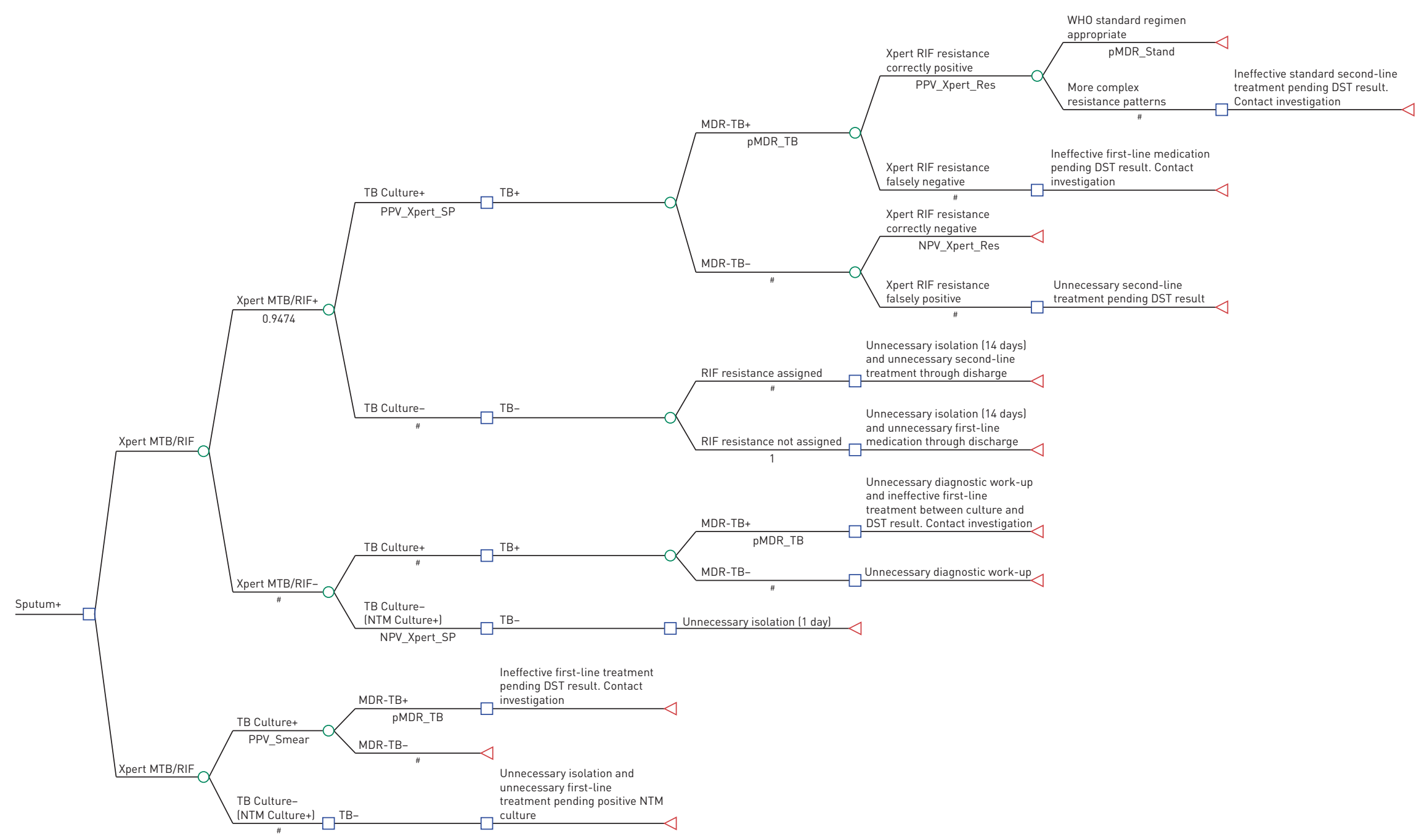

FIGURE 2 Xpert MTB/RIF versus the conventional approach in smear-positive multidrug-resistant tuberculosis (MDR-TB). NTM: nontuberculous mycobacteria; DST: drug susceptibility testing; RIF: rifampicin; WHO: World Health Organization. 


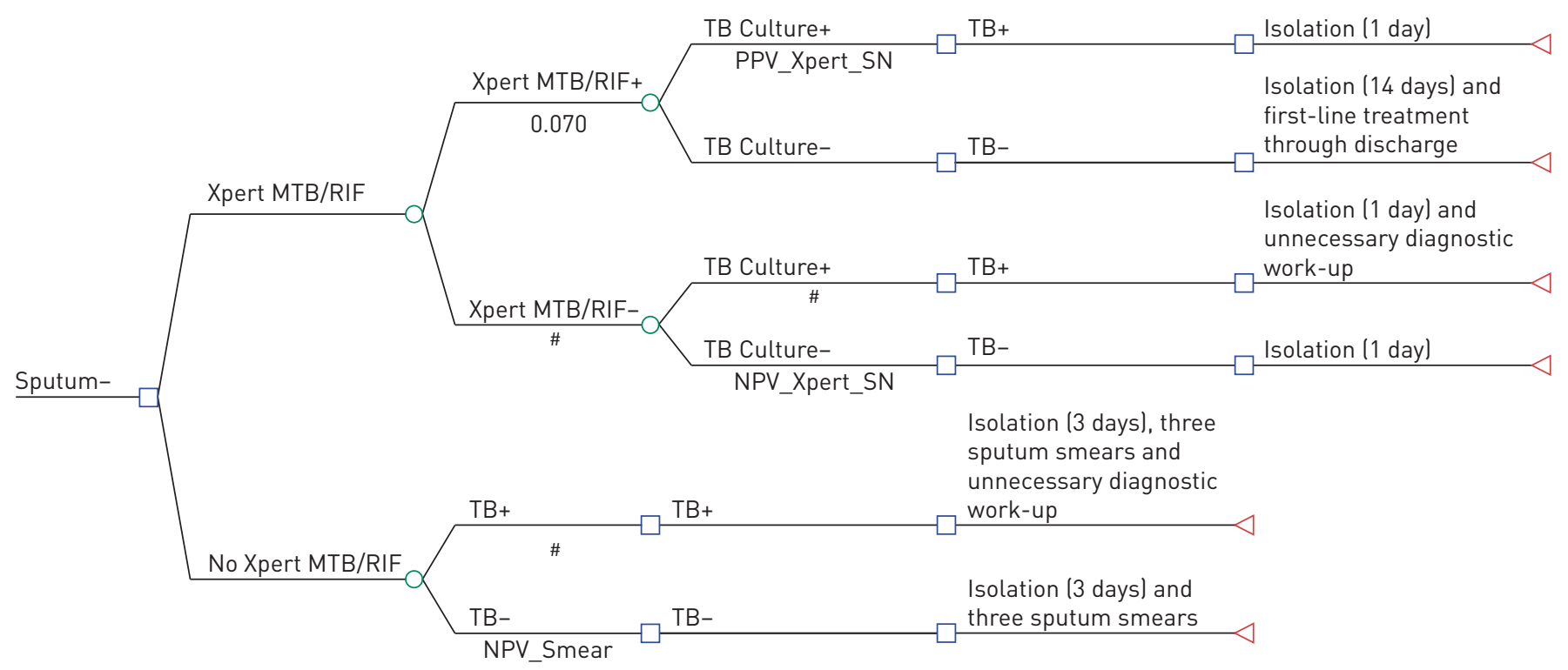

FIGURE 3 Xpert MTB/RIF versus the conventional approach in sputum-negative tuberculosis (TB) suspects.

95 specimens were smear-positive and 612 smear-negative compared with 133 specimens tested Xpert MTB/RIF-positive and 574 Xpert MTB/RIF-negative (table 1). Accuracy of microscopy and Xpert MTB/ RIF compared with culture results were calculated as well as PPV and NPV, and directly transferred as probabilities into the model. Of note, in our study the overall sensitivity (90.65\%; 95\% CI 84.54-94.93) and specificity (98.77; 95\% CI 97.48-99.50) of the Xpert MTB/RIF assay for the diagnosis of pulmonary TB was slightly higher than the pooled sensitivity of $88 \%$ (95\% CI $83-92$ ) and pooled specificity of $98 \%$ (95\% CI 97-99) reported in a recent Cochrane meta-analysis [13].

Based on the NRC data, the time (mean $\pm \mathrm{SD})$ to report positive cultures of $M$. tuberculosis among Xpert MTB/RIF-positives was 10.32 \pm 4.7 days (median 9 days, interquartile range (IQR) 7-11.75 days) for smear-positives and $13.17 \pm 5.15$ days (median 13 days, IQR 9-17 days) for smear-negatives (table 2). The time required from sample acquisition to DST results was registered; the waiting period was $23.9 \pm 8.9$ days

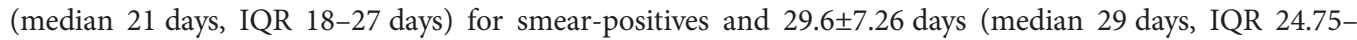
35 days) for smear-negatives.

In concurrence with the findings of STEINGART et al. [13], Xpert MTB/RIF was able to correctly distinguish between TB and nontuberculous mycobacteria (NTM) in smear-positive samples.

Calculation of the PPV and NPV of the Xpert MTB/RIF RIF resistance test follows the following definitions: $\mathrm{PPV}=$ sen $\times$ pre $/($ sen $\times$ pre $+(1-$ spe $) \times(1-$ pre $)) \quad$ and $\quad \mathrm{NPV}=$ spe $\times(1-$ pre $) /((1-$ sen $) \times$ pre + spe $\times(1-$ pre $))$, where sen=sensitivity, spe=specificity and pre=prevalence. According to a review by WeYER et al. [14], sensitivity and specificity of Xpert MTB/RIF for RIF resistance is $95 \%$ and $98 \%$, respectively, using culture as the reference method.

Although we assume the same prevalence for MDR-TB in our collective of MDR-TB suspects as for fully susceptible $\mathrm{TB}$ in the base case, that estimate is varied between $0 \%$ and $30 \%$ in our sensitivity analysis.

\section{Susceptibility of MDR-TB strains}

The determination of RIF resistance alone is not sufficient information for the establishment of case-appropriate therapy. The WHO standard concept for MDR-TB is effective in many RIF-resistant cases and it may be credibly started based on RIF resistance determination. However, DST must be performed in parallel and the therapy reconsidered once the full resistance pattern is known. If a change in therapy proves necessary, expensive but inappropriate treatment produces extraordinary costs as do the days spent with ineffective second-line treatment. In a recently published German cost analysis including the resistance patterns of MDR-TB strains [11], most strains $(51 / 55,92.72 \%)$ were in vitro susceptible to at least four drugs of the WHO standard scheme. We use that estimate as the base case value (pMDR_Stand).

\section{Economic parameters}

The analysis includes the drug and laboratory costs as well as the opportunity costs arising from revenue losses for the hospital (table 3): according to US and German guidelines [2, 15], sputum smear-positive 


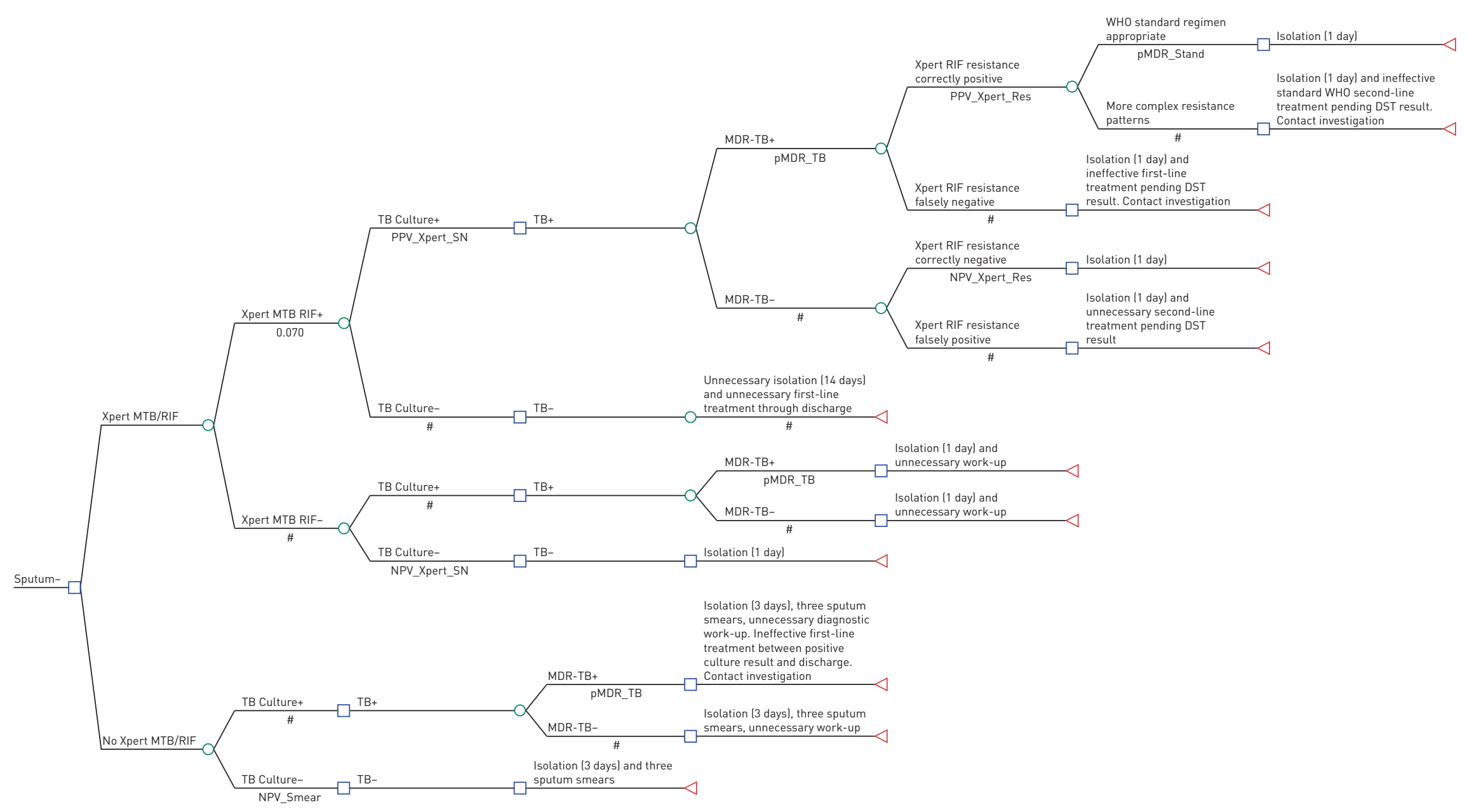

FIGURE 4 Xpert MTB/RIF versus the conventional approach in sputum-negative multidrug-resistant tuberculosis (MDR-TB) suspects. RIF: rifampicin; WHO: World Health Organization; DST: drug susceptibility testing. 


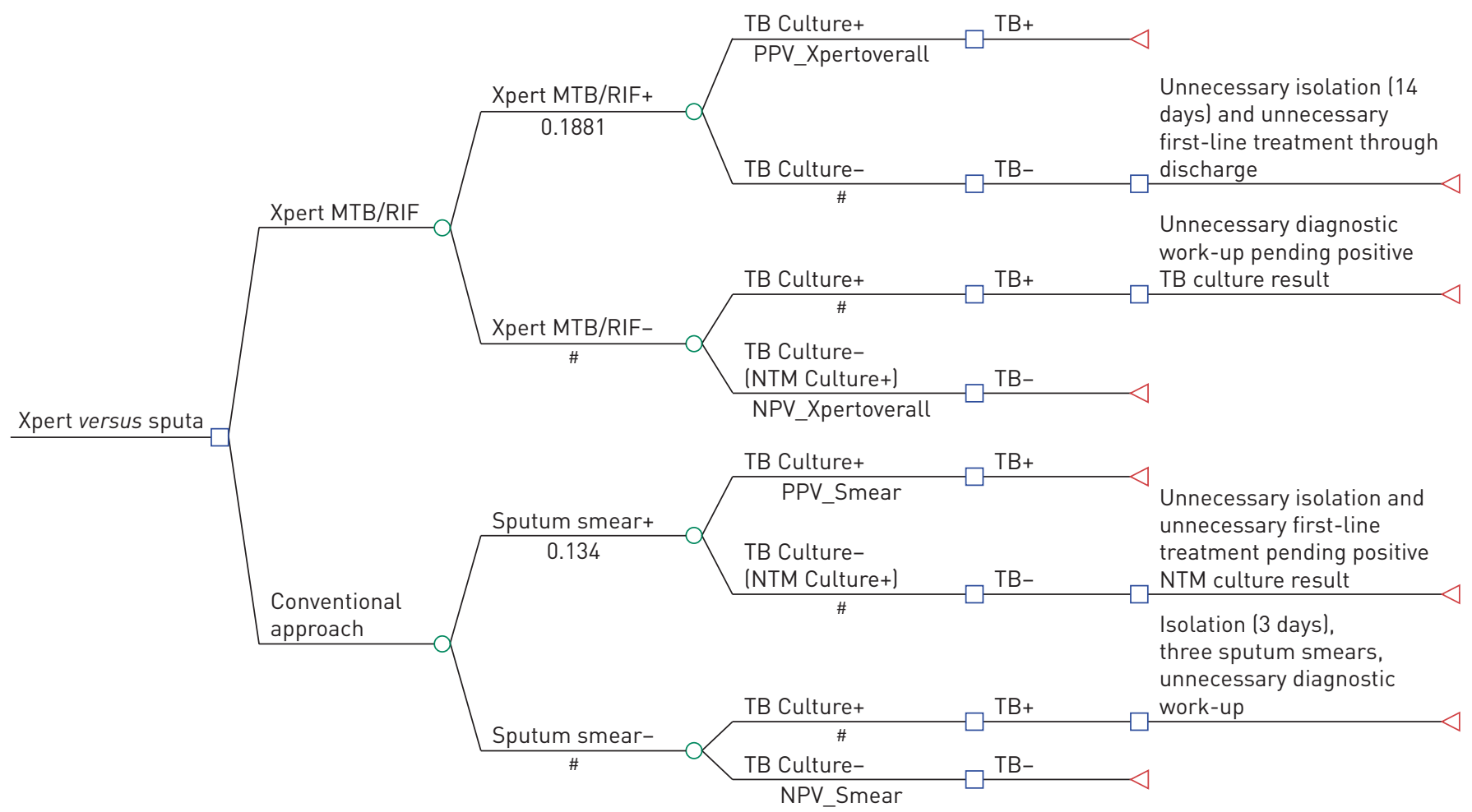

FIGURE 5 Xpert MTB/RIF versus the conventional approach in sputum-negative multidrug-resistant tuberculosis (MDR-TB) suspects. NTM: nontuberculous mycobacteria.

TB suspects must be isolated from the moment of admission through the first 14 days of treatment to counteract the nosocomial spread of TB, as must be smear-negative TB suspects until they have produced negative sputum samples on 3 subsequent days. Under the premise that most TB patients are accommodated in a twin-bedded room and that TB wards in Germany are working at full capacity, the loss of the use of one bed per day (cOpp) is incurred by the hospital during the isolation period.

The handling of smear-negative TB suspects or of those with a negative Xpert MTB/RIF result usually requires additional diagnostic work-up, the procedures and costs (cDcs) of which are presented in table 4.

In cases of unidentified MDR-TB, ineffective first-line treatment is administered either 1) following the conventional approach in the waiting period between positive TB culture and DST result or 2) in TB culture- and Xpert MTB/RIF-positives when no RIF resistance is assigned erroneously pending the DST result. Furthermore, ineffective second-line treatment is administered when the later DST result reveals more complex resistance patterns. Consequently, as in all of these cases when isolation is ended prematurely, intrahospital contact investigation must be performed (cContact). For full details and derivation of the cost parameters, see the online supplementary material.

\section{Results}

In base case analysis, performing Xpert MTB/RIF is cost-saving for every one of the five scenarios, when compared with the conventional procedure (sputum smear followed by culture), although to widely varying degrees (table 5).

Implementing Xpert MTB/RIF as an add-on in smear-positive TB suspects saves on average $€ 48.72$ per admitted patient. That this saving is relatively small is due to the fact that, according to our data, a positive TB culture result can be expected in as little as 10 days time; in non-TB smear-positives a NTM-positive culture result is also usually received. Thus, unnecessary isolation and unnecessary first-line treatment are promptly terminated. Potential cost savings strongly depend on the PPV of sputum smears and that of Xpert $\mathrm{MTB} / \mathrm{RIF}$ as well as on the actual delay until the sputum culture result can be received. Univariate sensitivity analysis, in which all variables in the decision trees receive assigned values within their respective ranges, reveals that due to the low proportion of NTM (5.3\%) in sputum smear-positives, even a minimal increase in the PPV of sputum smears (by $1.5 \%$ from $94.7 \%$ to $96.2 \%$ ) or a minimal decrease in the PPV of the Xpert MTB/RIF (by $1.1 \%$ to $98.9 \%$ ) results in a reversion of cost savings by utilising Xpert MTB/RIF as an add-on (online supplementary table S6a). The same is true if the waiting period for a positive culture result falls below a threshold of 7.5 days. 


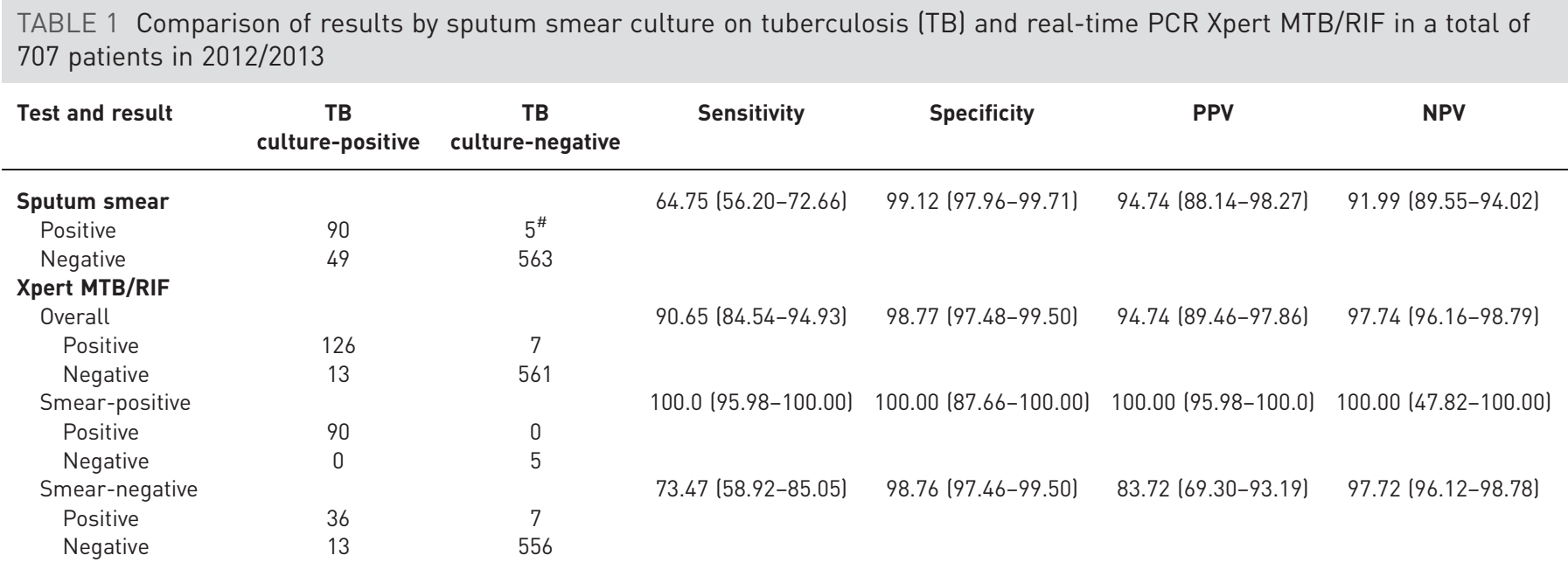

Data are presented as $\mathrm{n}$ or $\%(95 \% \mathrm{CI})$. NPV: negative predictive value; PPV: positive predictive value. ${ }^{\#}$ : samples from patients suffering from nontuberculous mycobacterial disease.

An increase in the cost of Xpert MTB/RIF by $20 \%$ reduces the average cost saving to a small total amount of only $€ 26.12$ and a $20 \%$ lower per-day cost of blocking a twin bed marginalises it to $€ 17.15$. Thus, in PSA with Monte-Carlo simulation of 1000 TB suspects, adding Xpert MTB/RIF in smear-positives suspected of having fully susceptible TB is less costly in only $46.4 \%$ of cases (online supplementary table S7).

In smear-positive MDR-TB cases, as compared with fully susceptible TB cases, additional cost savings may occur. These are attributable to the principal advantage of Xpert MTB/RIF of recognising RIF resistance and to the associated avoidance of ineffective first-line treatment during a wait for the result of a culture-based DST report. Furthermore, with Xpert MTB/RIF, a clearly lower number of contact individuals has to be screened for latent MDR-TB infection compared with the conventional approach, where MDR-TB may be spread to healthcare workers in the lag between termination of isolation after 14 days of ineffective treatment and the later DST report. Here, in the base case analysis, cost savings amount to $€ 189.56$ per person.

Depending on the PPV of assignment of RIF resistance by Xpert MTB/RIF, a variable percentage of cases receives ineffective, but costly second-line treatment, whilst the phenotypical DST result is initially lacking. MDR-TB prevalence strongly influences that PPV and consequently the amount of saving will be reduced to only $€ 48.30$ if MDR-TB prevalence goes to zero (online supplementary table S6b). Cost savings will also decrease in line with a decreasing number of contacts investigated and does not exceed $€ 105.44$ if only a total of five healthcare workers has to be screened, as compared with the 10 assumed in our base case (see online supplementary section 2.e).

In smear-positive MDR-TB cases, the probability that the WHO standard second-line treatment is applicable following the assignment of RIF resistance by Xpert MTB/RIF also plays a role. If the DST reveals more complex resistance strain patterns, cost savings in favour of Xpert MTB/RIF decrease remarkably to €79.08, assuming the standard regimen is effective in only $74 \%$ as a lower bound in univariate sensitivity analysis (online supplementary table S6b). PSA demonstrates that adding Xpert MTB/RIF in smear-positives suspected of having MDR-TB is less costly in $76.2 \%$ of cases (online supplementary table S7).

TABLE 2 Delays in reporting positive cultures and drug susceptibility testing results for Mycobacterium tuberculosis isolates separated by sputum smear status

Sputum smear-positive

$10.32 \pm 4.7(7-11.75)$

$23.9 \pm 8.9(8-27)$
Sputum smear-negative

$13.17 \pm 5.15(9-17)$

$29.6 \pm 7.26(24.75-35)$

Time to drug susceptibility testing report for isolates days

Data are presented as mean \pm SD (interquartile range). 


\begin{tabular}{|c|c|c|c|c|c|}
\hline Variables category & Variable name & Distribution ${ }^{\#}$ & $\begin{array}{l}\text { Value } \\
\text { (base case) }\end{array}$ & $\begin{array}{l}\text { Relative change } \\
\text { (range) }\end{array}$ & Reference \\
\hline Costs of first-line drugs per day $€$ & cTBD & Triangular & 6.3 & $\begin{array}{c} \pm 20 \% \\
(5.04-7.56)\end{array}$ & [6] \\
\hline Costs of WHO standard MDR-TB drugs per day $€$ & CMDR_TB & Triangular & 101.04 & $\begin{array}{c} \pm 20 \% \\
(80.83-121.25)\end{array}$ & [6] \\
\hline Costs of Ziehl-Neelsen microscopy $€$ & $c Z N$ & Triangular & 6.41 & $\begin{array}{l} \pm 20 \% \\
(5.12-7.69)\end{array}$ & GOÄ no. 4513 (online supplementary section 2.b) \\
\hline Costs of mycobacterial culture $€$ & cCulture & Triangular & 23.31 & $\begin{array}{c} \pm 20 \% \\
(18.65-27.97)\end{array}$ & G0Ä no. 4540 (online supplementary section 2.b) \\
\hline Costs of Xpert MTB/RIF $€$ & cXpert & Triangular & 110.75 & $\begin{array}{c} \pm 20 \% \\
(88.6-130.9]\end{array}$ & $\begin{array}{c}\text { GOÄ nos. } 4780 \text { and } 4784 \text { (online supplementary } \\
\text { section 2.b) }\end{array}$ \\
\hline Opportunity costs of blocking twin bed $€$ & cOpp & Triangular & 314.71 & $\begin{array}{c} \pm 20 \% \\
(253.97-380.95)\end{array}$ & $\begin{array}{l}\text { Calculated from InEK (http://www.g-drg.de/cms/) } \\
\text { data (online supplementary section 2.a) }\end{array}$ \\
\hline Costs of diagnostic work-up $€$ & cDcs & Triangular & 306.81 & $\begin{array}{c} \pm 20 \% \\
(245.45-368.17)\end{array}$ & Table 4 (online supplementary section 2.d) \\
\hline $\begin{array}{l}\text { Latency pending sputum culture result in } \\
\text { smear-positive day }\end{array}$ & dCulture_SP & Normal & 10.32 & $\begin{array}{l} \pm S D 8.9 \\
(5.62-15.02)\end{array}$ & Assessed (table 2) \\
\hline Latency pending DST result in smear-positives day & dResistance_SP & Normal & 23.9 & $\begin{array}{c} \pm \text { SD } 8.9 \\
(15.0-32.8)\end{array}$ & Assessed (table 2) \\
\hline $\begin{array}{l}\text { Latency pending sputum culture result in } \\
\text { smear-negatives day }\end{array}$ & dCulture_SN & Normal & 13.17 & $\begin{array}{c} \pm \text { SD } 5.15 \\
(8.02-18.32)\end{array}$ & Assessed (table 2) \\
\hline Latency pending DST result in smear-negatives day & dResistance_SN & Normal & 29.2 & 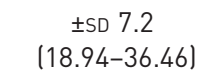 & Assessed (table 2) \\
\hline Probability of MDR-TB in TB patients & pMDR_TB & Linear & 0.1966 & $\begin{array}{l}0 \% / 30 \% \\
(0.0-0.3)\end{array}$ & Calculated (table 1) \\
\hline PPV of positive sputum smear & PPV_Smear & Linear & 0.9474 & $\begin{array}{c} \pm 95 \% \mathrm{Cl} \\
(0.8814-0.9827)\end{array}$ & Calculated (table 1) \\
\hline NPV of negative sputum smear & NPV_Smear & Linear & 0.9199 & $\begin{array}{c} \pm 95 \% \mathrm{Cl} \\
(0.8955-0.9402)\end{array}$ & Calculated (table 1) \\
\hline PPV of Xpert MTB/RIF in smear-positives & PPV_Xpert_SP & Linear & 1.0 & $\begin{array}{c} \pm 95 \% \mathrm{Cl} \\
(0.9598-1.0)\end{array}$ & Calculated (table 1) \\
\hline PPV of Xpert MTB/RIF in smear-negatives & PPV_Xpert_SN & Linear & 0.8372 & $\begin{array}{c} \pm 95 \% \mathrm{Cl} \\
(0.6930-0.9319)\end{array}$ & Calculated (table 1) \\
\hline NPV of Xpert MTB/RIF in smear-positives & NPV_Xpert_SP & Linear & 1.0 & $\begin{array}{c} \pm 95 \% \mathrm{Cl} \\
(0.4782-1.0)\end{array}$ & Calculated (table 1) \\
\hline NPV of Xpert MTB/RIF in smear-negatives & NPV_Xpert_SN & Linear & 0.9772 & $\begin{array}{c} \pm 95 \% \mathrm{Cl} \\
(0.9623-0.9878)\end{array}$ & Calculated (table 1) \\
\hline
\end{tabular}




\begin{tabular}{|c|c|c|c|c|c|}
\hline Variables category & Variable name & Distribution ${ }^{\#}$ & $\begin{array}{c}\text { Value } \\
\text { (base case) }\end{array}$ & $\begin{array}{l}\text { Relative change } \\
\text { (range) }\end{array}$ & Reference \\
\hline PPV of Xpert MTB/RIF irrespective of smear status & PPV_Xpertoverall & Linear & 0.9474 & $\begin{array}{c} \pm 95 \% \mathrm{Cl} \\
(0.8946-0.9786)\end{array}$ & Calculated (table 1) \\
\hline NPV of Xpert MTB/RIF irrespective of smear status & NPV_Xpertoverall & Linear & 0.9747 & $\begin{array}{c} \pm 95 \% \mathrm{Cl} \\
(0.9612-0.9879)\end{array}$ & Calculated (table 1) \\
\hline Probability that WHO standard regimen is effective & pMDR_Stand & Linear & 0.9272 & $\begin{array}{c} \pm 20 \% \\
(0.741-1)\end{array}$ & [11] \\
\hline Time left to discharge from hospital days & dDischarge & Triangular & 26.78 & $\begin{array}{c} \pm 20 \% \\
(21.24-32.14)\end{array}$ & [6] \\
\hline $\begin{array}{l}\text { Costs of intrahospital contact investigation per TB } \\
\text { index case } €\end{array}$ & cContact & Triangular & 105.81 & $\begin{array}{c} \pm 20 \% \\
(84.65-126.97)\end{array}$ & Adapted from [12] \\
\hline Number of contacts to be investigated & pContact & Triangular & 10 & $5-20$ & Assumption (online supplementary section 2.e) \\
\hline
\end{tabular}


TABLE 4 Costs of diagnostic work-up in sputum smear-negative or Xpert MTB/RIF-negative tuberculosis suspects

\begin{tabular}{lcc} 
Procedure & $\mathbf{n}$ & GOÄ (1.0 times rate) $€$ \\
\hline Computed tomography thorax & 5371 & 134.06 \\
Contrast agent, injection intravenously, high pressure & 346 & 17.49 \\
Bronchoscopy including lavage & 678 & 52.46 \\
Cytological investigation & 4852 & 10.14 \\
Interferon- $\gamma$ release assay test peripheral blood & 3694 & 33.80 \\
Ziehl-Neelsen smear (lavage) & 4513 & 9.33 \\
PCR (lavage) & 4783 & 29.41 \\
Culture mycobacteria (lavage) & 4540 & 33.31 \\
Total & & 306.81
\end{tabular}

GOÄ: Gebührenordnung für Ärzte (German medical fee schedule).

In contrast, and predominantly due to a shorter isolation period ( 2 days fewer blockage of a twin bed by using Xpert MTB/RIF), the cost saving in fully susceptible smear-negative TB suspects is $€ 503$ and remains constantly high, even when the main cost driver, the per-day hospital opportunity cost of blocking a twin bed, is assumed to be $20 \%$ lower (in this case reducing the saving to $€ 385.42$ ). Increasing the cost of Xpert MTB/RIF within its given range or decreasing the higher NPV that its $9 \%$ sensitivity advantage over sputum smear brings ( $73.47 \%$ versus $64.75 \%)$ diminishes the total amount of savings at most by $8.44 \%$ and $1.24 \%$, respectively, and has only marginal impact (online supplementary table S6c).

For smear-negative MDR-TB cases, the figure for the cost saving is slightly higher at $€ 515.25$ because, as is the case in smear-positive MDR-TB patients, it is predominantly the costs of contact investigation of healthcare workers that have to considered. Due to the high NPV (92\%) of sputum smears, however, only very few MDR-TB cases will be detected in smear-negatives which induce hospital contact investigations by possible transmission in the latency between positive culture and the later DST result. Therefore, neither variations of MDR-TB prevalence nor of the number of contact individuals in sensitivity analysis have a substantial impact on cost savings (online supplementary table S6d) and in PSA cost savings remain at $100 \%$ in all scenarios involving smear-negative TB or MDR-TB suspects (online supplementary table S7).

Full replacement of the conventional approach by Xpert MTB/RIF saves $€ 449.98$. This is primarily due to the elimination of unnecessary isolation in the $87 \%$ smear-negatives of our cohort of TB suspects (612/707; table 1). Accordingly, reducing the figure of per-day opportunity cost to its lower bound $(-20 \%)$ will reduce the amount of cost saving by $59 \%$ to only $€ 184.67$ (online supplementary table S6e).

TABLE 5 Results of base case analysis (five scenarios)

\begin{tabular}{|c|c|c|}
\hline Base case analysis and comparator & Mean cost per patient & Incremental cost ${ }^{\#}$ \\
\hline \multicolumn{3}{|l|}{ Sputum smear-positive TB suspects } \\
\hline Xpert MTB/RIF as an add-on & 157.2 & 0 \\
\hline Conventional approach & 205.47 & 48.27 \\
\hline \multicolumn{3}{|c|}{ Sputum smear-positive MDR-TB suspects } \\
\hline Xpert MTB/RIF as an add-on & 240.93 & 0 \\
\hline Conventional approach & 430.49 & 189.56 \\
\hline \multicolumn{3}{|l|}{ Sputum smear-negative TB suspects } \\
\hline Xpert MTB/RIF as an add-on & 512.17 & 0 \\
\hline Conventional approach & 1015.17 & 503.0 \\
\hline \multicolumn{3}{|c|}{ Sputum smear-negative MDR-TB suspects } \\
\hline Xpert MTB/RIF as an add-on & 518.03 & 0 \\
\hline Conventional approach & 1033.28 & 515.25 \\
\hline \multicolumn{3}{|l|}{ Xpert MTB/RIF replacing smears } \\
\hline Xpert MTB/RIF & 440.97 & 0 \\
\hline Conventional approach & 890.95 & 449.98 \\
\hline \multicolumn{3}{|c|}{$\begin{array}{l}\text { Costs are presented as } € \text {. TB: tuberculosis; MDR: multidrug-resistant. \#: increase in total costs resulting } \\
\text { from using the conventional approach alone versus including Xpert MTB/RIF as an add-on or as a } \\
\text { replacement. }\end{array}$} \\
\hline
\end{tabular}




\section{Discussion}

The present study is a differentiated cost-benefit analysis of the implementation of the real-time PCR Xpert MTB/RIF method in hospitalised patients with suspected TB, either as an adjunct to or a replacement for sputum smear microscopy. PCR, with its ability to very rapidly confirm or exclude infectious pulmonary $\mathrm{TB}$, has the potential to minimise the duration of isolation and/or to avoid unnecessary isolation.

To date, only very few cost studies on the routine use of nucleic acid amplification tests have been published and their findings are unfortunately not applicable to German conditions. AdeLmanN et al. [16] found significant cost savings in a US urban public hospital (US\$2003 per suspected smear-positive TB case) when using the amplified MDT (Mycobacterium tuberculosis Direct) test (Gen-Probe, San Diego, CA, USA) among predominantly African-American AFB smear-positive TB suspects. These subjects had a high prevalence of HIV-1 infection and for their cases the AFB smear had a very low PPV (27\%) for culture-confirmed TB. Germany's HIV prevalence is low (2009: 0.1\% of individuals aged 15-49 [17]) and the data we used from the German NRC give the AFB smear a PPV for culture-confirmed TB of 95\%. In Adelmann et al. [16], one main cost driver was the cost of unnecessary contact investigations, which were begun not at the time of culture confirmation, but immediately upon recognition of the positive AFB smear. A second notable driver was the per-day cost difference between isolation and nonisolation rooms. In Germany, special airborne infection isolation rooms providing negative pressure are rare. Also in Millmann et al.'s cost-benefit analysis [18], the incremental cost of respiratory isolation per day in a special room and the reduction regarding the length of stay of on average from 2.7 to 1.4 days per patient saved US\$2278 per admission of suspected pulmonary TB. Our hospital costs are significantly lower.

Our analysis shows that in base case analysis, performing Xpert MTB/RIF as an add-on for TB suspects admitted to a German TB ward as well as complete replacement of sputum smears by Xpert MTB/RIF is consistently cost-saving, even when the economic perspective is restricted to hospitalisation. Driving this is a reduction in the number of isolation days per case, each of which results in a blocked twin bed and corresponding revenue loss for the hospital. Smear-positive and smear-negative patients on average wait 10 and 13 days, respectively, for their positive culture results in Germany; for phenotypical DST results the average waiting periods are 24 and 30 days. There is only a marginal difference of specificity and overlapping 95\% confidence interval in the PPV of fully susceptible smear-positive TB patients. This is due to a low observed proportion of $5.3 \%$ of NTM, resulting, as sensitivity analysis reveals, in a fragile economic advantage for Xpert MTB/RIF in this group of patients.

Paradoxically, and counter-intuitively, one strikingly favourable feature of Xpert MTB/RIF has its drawbacks. The immediate detection of RIF resistance in MDR-TB patients, in comparison with the much later reportable, culture-based DST, brings additional costs that occur in those patients with complex resistance patterns and for which the preliminary treatment with the recommended WHO standard second-line treatment is not effective. The treatment, ongoing for a number of weeks, must be adjusted to correspond with the pattern identified by phenotypical DST. In these cases, treatment duration is ultimately as long as it would have been under the conventional approach. However, DST cannot fully be replaced by line probe assays as the sensitivity of these for resistance to ethambutol, ofloxacin and injectable drugs is limited even in smear-positives [19]. Even in the case of a positive resistance determination, susceptibility to the other drugs must be clarified by DST in order to implement a definitely appropriate therapeutic regimen.

Another drawback of RIF detection by Xpert MTB/RIF is that unnecessary second-line treatment will be prescribed in a number of cases that corresponds to $1-\mathrm{PPV}$ of the test. This rate of false resistance determinations logically increases with decreasing MDR-TB prevalence.

PSA that considers all realistic assumptions of uncertainty confirms the different degrees of potential savings between the five scenarios and underlines that Xpert MTB/RIF is most likely to be cost-saving in smear-negative TB suspects. This is of particular relevance as in 2013 in Germany 79.6\% (2624/3298) of all reported pulmonary TB cases were "open" (culture-confirmed), of which 55\% (1443/2624) were sputum smear-negative, and also in pulmonary MDR-TB cases only 63.5\% (54/85) were sputum smear-positive (B. Brodhun, Robert Koch Institute, personal communication, 2015).

Our study also has some limitations that must be considered when interpreting our results. First, the general limitations of retrospective, single-centre studies have to be considered. Our use of extensive sensitivity analyses is an effort that addresses those limitations. To validate our estimates, more cost studies, preferably with a multicenter and prospective study design, are required.

Second, from an economic point of view, replacing smear examination by Xpert MTB/RIF rather than using it as an add-on option basically combines the advantages of implementing Xpert MTB/RIF for the 
single smear-positive or smear-negative categories. However, it must be emphasised that graduated contact tracing according to the degree of infectiousness of the index case $[2,15,20]$, as is usually practiced in low-incidence countries, could no longer be done were sputum examination to lapse. In practice, then, as all index cases have to be considered potentially sputum-positives, the initial circle of contact individuals to be investigated by the public health departments would have to be drastically expanded to be on the safe side, resulting in an inestimable increase in total costs.

\section{Conclusion}

The utilisation of Xpert MTB/RIF in Germany, a high-income country, is likely to reduce overall costs in cases of suspected TB, especially in MDR-TB and smear-negative patients. As such, routine use of Xpert MTB/RIF may have a direct and positive impact on the control of TB disease. Prospective clinical studies should be undertaken to further evaluate its economic advantages in the immediate future.

\section{References}

1 European Centre for Disease Prevention and Control/WHO Regional Office for Europe. Tuberculosis surveillance and monitoring in Europe 2014. Stockholm, European Centre for Disease Prevention and Control, 2014.

2 Centers for Disease Control and Prevention. Guidelines for preventing the transmission of Mycobacterium tuberculosis in health-care settings, 2005. MMWR Recomm Rep 2005; 54: RR-17, 1-141.

3 Migliori GB, Sotgiu G. Assessing tuberculosis management: what really happens to patients? Lancet Infect Dis 2015; 15: 1249-1251.

4 Lönnroth K, Migliori GB, Abubakar I, et al. Towards tuberculosis elimination: an action framework for low-incidence countries. Eur Respir J 2015; 45: 928-952.

5 Vogl M. Assessing the DRG cost accounting with respect to resource allocation and tariff calculation: the case of Germany. Health Econ Rev 2012; 2: 15.

6 Diel R, Rutz S, Castell S, et al. Tuberculosis: cost of illness in Germany. Eur Respir J 2012; 40: 143-151.

7 Raizada N, Sachdeva KS, Sreenivas A, et al. Feasibility of decentralised deployment of XPERT MTB/RIF test at lower level of health system in India. PLoS One 2014; 9: e89301.

8 WHO. Automated real-time nucleic acid amplification technology for rapid and simultaneous detection of tuberculosis and rifampicin resistance: Xpert MTB/RIF system for the diagnosis of pulmonary and extrapulmonary tuberculosis in adults and children. http://apps.who.int/iris/handle/10665/112472 Date last accessed: August 5, 2015.

9 US Food and Drug Administration. Decision summary. https://www.accessdata.fda.gov/scripts/cdrh/cfdocs/cfPMN/ denovo.cfm?ID=DEN130032 Date last accessed: August 5, 2015.

10 Robert Koch-Institut. Bericht zur Epidemiologie der Tuberkulose in 2012 [Report on the epidemiology of tuberculosis in Germany in 2013]. Berlin, Robert Koch-Institut, 2014.

11 Diel R, Nienhaus A, Lampenius N, et al. Cost of multidrug resistance tuberculosis in Germany. Respir Med 2014; 108: 1677-1687.

12 Diel R, Nienhaus A, Lange C, et al. Cost-optimisation of screening for latent tuberculosis in close contacts. Eur Respir J 2006; 28: 35-44.

13 Steingart KR, Sohn H, Schiller I, et al. XPERT ${ }^{\infty}$ MTB/RIF assay for pulmonary tuberculosis and rifampicin resistance in adults. Cochrane Database Syst Rev 2013; 1: CD009593.

14 Weyer K, Mirzayev F, Migliori GB, et al. Rapid molecular TB diagnosis: evidence, policy making and global implementation of Xpert MTB/RIF. Eur Respir J 2013; 42: 252-271.

15 Ziegler R, Just HM, Castell S, et al. Tuberculosis infection control - recommendations of the DZK. Gesundheitswesen 2012; 74: 337-350.

16 Adelman MW, Kurbatova E, Wang YF, et al. Cost analysis of a nucleic acid amplification test in the diagnosis of pulmonary tuberculosis at an urban hospital with a high prevalence of TB/HIV. PLoS One 2014; 9: e100649.

17 Trading Economics. Prevalence of HIV - total (\% of population ages 15-49) in Germany. www.tradingeconomics. com/germany/prevalence-of-hiv-total-percent-of population-ages-15-49-wb-data.html Date last accessed: August 5, 2015.

18 Millman AJ, Dowdy DW, Miller CR, et al. Rapid molecular testing for TB to guide respiratory isolation in the U.S.: a cost-benefit analysis. PLoS One 2013; 8: e79669.

19 Theron G, Peter J, Richardson M, et al. The diagnostic accuracy of the GenoType MTBDRsl assay for the detection of resistance to second-line anti-tuberculosis drugs. Cochrane Database Syst Rev 2014; 10: CD010705.

20 Diel R, Loytved G, Nienhaus A, et al. New recommendations for contact tracing in tuberculosis. German Central Committee against Tuberculosis. Pneumologie 2011; 65: 359-378. 